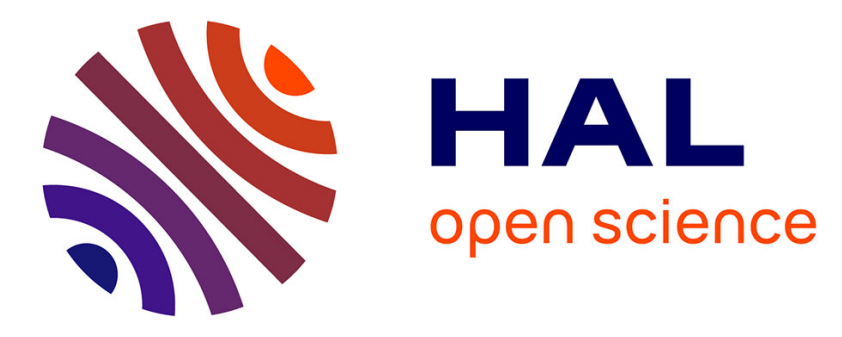

\title{
Weak polyelectrolyte brushes: re-entrant swelling and self-organization
}

\author{
Vincent Senechal, Hassan Saadaoui, Nelson Vargas-Alfredo, Juan \\ Rodriguez-Hernandez, Carlos Drummond
}

\section{- To cite this version:}

Vincent Senechal, Hassan Saadaoui, Nelson Vargas-Alfredo, Juan Rodriguez-Hernandez, Carlos Drummond. Weak polyelectrolyte brushes: re-entrant swelling and self-organization. Soft Matter, 2020, 16 (33), pp.7727-7738. 10.1039/d0sm00810a . hal-02926847

\section{HAL Id: hal-02926847 https://hal.science/hal-02926847}

Submitted on 1 Sep 2020

HAL is a multi-disciplinary open access archive for the deposit and dissemination of scientific research documents, whether they are published or not. The documents may come from teaching and research institutions in France or abroad, or from public or private research centers.
L'archive ouverte pluridisciplinaire HAL, est destinée au dépôt et à la diffusion de documents scientifiques de niveau recherche, publiés ou non, émanant des établissements d'enseignement et de recherche français ou étrangers, des laboratoires publics ou privés. 


\section{Weak Polyelectrolyte Brushes: Re-entrant Swelling and Self- Organization}

Received 00th January 20xx, Accepted 00th January 20xx

\begin{abstract}
Vincent Senechal, ${ }^{a, b}$ Hassan Saadaoui, ${ }^{a, b}$ Nelson Vargas-Alfredoc ${ }^{c}$ Juan Rodriguez-Hernandez ${ }^{c}$ and Carlos Drummond ${ }^{a, b}$ * $^{*}$
\end{abstract}

\section{Introduction}

End-grafted polymers (polymer brushes) have been the subject of intense studies during the last few decades, mainly driven by their potential for the control of surface properties, ranging from protective coatings and biofouling to control of adhesion and lubrication. ${ }^{1}$ These properties are often determined by the configuration of the grafted macromolecules, which depend, in turn, on the physicochemical environment; this reversible response makes polymer coatings good ingredients for the design of stimuli-responsive ('smart') materials., ${ }^{1,2,3,4}$ In particular, polyelectrolyte coatings enable the control of surface properties by changing salinity or applying external fields. Weak (annealed) polyelectrolytes are more versatile than strong polyions, as their degree of ionization is also sensitive to the physicochemical environment (i.e. solvent quality, $\mathrm{pH}$, ionic strength). Thus, charge regulation plays a dominant role in the properties polyacids or polybases. For the case of polyacids, the degree of ionization is determined by the equilibrium between charged and uncharged monomers regulated by the $\mathrm{pH}$, given by:

$$
\mathrm{AH}+\mathrm{H}_{2} \mathrm{O} \leftrightarrow A^{-}+\mathrm{H}_{3} \mathrm{O}^{+} \quad K_{a}=\frac{\left[\mathrm{H}_{3} \mathrm{O}^{+}\right]\left[\mathrm{A}^{-}\right]}{[A H]}
$$

\footnotetext{
a. CNRS, Centre de Recherche Paul Pascal (CRPP), UMR 5031, F-33600 Pessac, France.

b. Université de Bordeaux, Centre de Recherche Paul Pascal. F- 33600 Pessac, France

c. Instituto de Ciencia y Tecnología de Polímeros, CSIC, Juan de la Cierva 3, 28006, Madrid, Spain.

+ Electronic Supplementary Information (ESI) available: [details of any supplementary information available should be included here]. See
} DOI: $10.1039 / x 0 x x 00000 x$ where $K_{a}$ is the monomer dissociation constant. $K_{a}$ may depend on the position of the monomer in the chain, and it is not necessarily the same as to the one of monomers in solution. ${ }^{5}$ The situation is even more complex for grafted chains: in this case the ionization will be controlled by the $\mathrm{pH}$ inside the brush (which may be different from the bulk $\mathrm{pH}$ ) due to the influence of the charged boundary. The difference between local and global $\mathrm{pH}$ may be particularly important at low ionic strengths. In the absence of added salt (i.e., absence of indifferent counterion), protons released as consequence of monomer ionization will remain inside/close to the grafted polymer chains, to assure local electroneutrality. As a consequence, local $\mathrm{pH}$ is largely decreased compared to bulk $\mathrm{pH}$, hindering the ionization of the polyions (eq. 1). Regarding this point, Klein and coworkers discussed how the titration of a polyacid can be very different in the absence of added salt (with the ionic strength determined by $\mathrm{pH}$ ) or adjusting the ionic strength by the addition of a supporting electrolyte. ${ }^{6}$

The response of polyelectrolyte brushes to a changing environment has been addressed by a large number of studies. Nevertheless, some features are still not fully described. In particular, a thorough description of the possibility of inhomogeneous density in the brush is still emerging. An overview on this subject was recently reported by Ballauff and Borisov. ${ }^{7}$ A distinction can be made between normal and inplane heterogeneity. Theoretical and numerical studies have predicted the possibility of inhomogeneity along the normal direction in weak polyion brushes.8,9,10 Concentrationdependent solvent quality has been shown to induce vertical phase segregation in polymer brushes. ${ }^{11}$ On the contrary, experimental evidences for this scenario are rather limited. ${ }^{12,13,14}$ 
Regarding in-plane self-organization, a number of theoretical studies have addressed the issue of localized aggregation in polymer brushes, which result of the interplay between shifting chemical equilibrium (for weak polyelectrolytes), physical interactions, and the limited mobility of the grafted chains. Few decades ago, scaling analysis was used to show that neutral polymer brushes at low grafting density can form pinned micelles under low solvent quality. ${ }^{15,16}$ In a study of neutral polymer brushes in poor solvent combining Monte Carlo simulations and scaling analysis, Pattanayek and coworkers ${ }^{17}$ predicted the formation different surface aggregate including micelles, holes and lamellar morphology, depending on grafting density or chain length. Similarly, chain clustering in bad solvent conditions has been predicted for the case of polyelectrolytes for degrees of ionization below a threshold value. ${ }^{18}$ Combining molecular dynamic simulations and scaling analysis, Dobrynin and coworkers demonstrated the possibility of formation of cylindrical or lamellar polyelectrolyte chain aggregates in conditions of poor solvent for the polymeric backbone. ${ }^{19,20}$ Szleifer and coworkers developed a molecular theory which included local chemical equilibrium and physical interactions to describe weak polyelectrolyte brushes. They observed that the competition between the different interactions can promote self-assembly of different morphologies, including pinned spherical or cylindrical micelles, holes, uniform layer, or phase coexistence. ${ }^{21,22}$ On the contrary, experimental reports on micro-structured solvated polymer brushes are rather scarce. By using atomic force microscopy, Hadziioannou and coworkers observed the formation of pinned micelles in polystyrene PS brushes immersed in water. ${ }^{23}$ Reversible microphase separation of PS in cyclohexane at temperature below the theta temperature has also been reported. ${ }^{24}$ Choi and co-workers reported the formation of micellar aggregates in a brush of thermo-responsive poly(Nisopropyl acrylamide) at low grafting densities and above the critical solution temperature (poor solvent condition). ${ }^{25}$ By using X-ray reflectivity, Gunther and coworkers observed chain aggregation in a strong polyelectrolyte brush under high salinity conditions, which favored the short range attraction over the interchain electrostatic repulsion. ${ }^{26}$ In a study of weak polyeletrolyte brushes Hur and co-workers observed polymer aggregation and microphase coexistence (smooth/structured) depending on ionic strength. ${ }^{27}$

In this work we investigated the response of polyion brushes of an annealing polyelectrolyte (polyacrylic acid) to a changing environment, by combining Quartz Crystal Microbalance (QCM) and Atomic Force Microscopy (AFM). We explored conditions of relatively low grafting density; our aim was to study the structure and responsiveness of this system close to the mushroom-brush transition, a cross-over regime which combines substantial surface coverage with maximal polymer responsiveness, due to limited steric hindrance to conformational changes in grafted chains. ${ }^{28}$

\section{Experimental}

Materials and methods
Styrene (St) (Aldrich, 99\%), tert-butyl acrylate (tBA) (SigmaAldrich, 98\%) were distilled under reduced pressure over calcium hydride prior to use. Copper (I) bromide (CuBr) (SigmaAldrich, 98\%), 2,2'-bipyridyl (bipy) (Sigma-Aldrich, 99+\%), $\mathrm{N}, \mathrm{N}, \mathrm{N}, \mathrm{N}^{\prime}, \mathrm{N}$ ",-pentamethyldiethylenetriamine (PMDETA) (Sigma-Aldrich, 99\%), phenylethyl bromide (PhEBr) (SigmaAldrich, 97\%) and other solvents were used as received.

Polystyrene-block-poly(acrylic acid) (PS-b-PAA) was prepared by controlled Atom Transfer Radical Polymerization (ATRP) in two steps, as we have reported before. ${ }^{4}$ First, a polystyrene macroinitiator was prepared, followed by the synthesis of PS-bPtBA. Finally, the PS-b-PAA copolymers were obtained by hydrolysis of the PtBA block. A copolymer $\mathrm{PS}_{36}-\mathrm{b}-\mathrm{PAA}_{125}$, with $P D=1.45$ was used for all the results presented in this work. Precise details of the methods of synthesis, purification and characterization have been reported before. ${ }^{4}$

Disulfide functionalized poly(acrylic acid) $\left(\mathrm{PAA}_{47}\right)_{2} \mathrm{~S}_{2}$ was prepared by deprotection of the tert-butyl groups from Poly(tert-butyl acrylate disulfide $\left(\mathrm{PtBA}_{47}\right)_{2} \mathrm{~S}_{2}$. The preparation of $\left(\mathrm{PtBA}_{47}\right)_{2} \mathrm{~S}_{2}$ was carried out as follows: $0.026 \mathrm{~g}(0.18 \mathrm{mmol})$ of $\mathrm{Cu}(\mathrm{I}) \mathrm{Br}$ was placed in a Schlenk tube. Next $2.207 \mathrm{~g}(17.22 \mathrm{mmol})$ of deoxygenated tert-butyl acrylate (tBA), $0.041 \mathrm{~g}(0.24 \mathrm{mmol})$ of $\mathrm{N}, \mathrm{N}, \mathrm{N}^{\prime}, \mathrm{N}^{\prime \prime}, \mathrm{N}^{\prime \prime}$-pentamethyldiethylenetriamine (PMDETA), $0.054 \mathrm{~g}(0.12 \mathrm{mmol})$ of bis [2-(2'-bromoisobutyryloxy) ethyl] disulfide (BiBOE) ${ }_{2} \mathrm{~S}_{2}$ and $1.5 \mathrm{ml}$ of deoxygenated acetone ( $5: 3$ by volume monomer/solvent) were added to the tube. All the reactants were added under nitrogen flow. The reaction was carried out under nitrogen atmosphere at $50{ }^{\circ} \mathrm{C}$ during $6 \mathrm{~h}$. Then, the reaction mixture was diluted with chloroform and passed through an alumina column to eliminate the copper catalyst. The polymer was precipitated with methanol, dried and characterized by GPC and ${ }^{1} \mathrm{H}$ NMR. To deprotect the $\left(\mathrm{PtBA}_{47}\right)_{2} \mathrm{~S}_{2}$ and obtain the desired $\left(\mathrm{PAA}_{47}\right)_{2} \mathrm{~S}_{2}, 0.5 \mathrm{~g}$ of poly tertbutyl acrylate disulfide $\left(\mathrm{PtBA}_{47}\right)_{2} \mathrm{~S}_{2}$ were diluted in dichloromethane $\left(\mathrm{CH}_{2} \mathrm{Cl}_{2}\right)$ in a round flask. Then, $2.23 \mathrm{~g}(19.52$ $\mathrm{mmol}$ ) of trifluoroacetic acid were added, and the mixture was stirred during $72 \mathrm{~h}$ at room temperature, forming a white powder precipitate. The solvent was removed by filtration and the product was washed five times with dichloromethane to eliminate the acid residues.

Polymer brush assembly. Two methods were used to produce poly(acrylic acid) brushes. First, we used the Langmuir-Schaefer approach, as described by Currie and coworkers, ${ }^{29,30}$ and used by us in previous studies. 4,31 In brief, QCM crystals with gold electrodes were coated by $50 \mathrm{~nm}$ thin polystyrene PS (Acros Organics, Mw 250kDa) films by spin coating, which were annealed overnight at $95^{\circ} \mathrm{C}$ before use. To deposit the copolymer, $5 \mathrm{mg}$ of $\mathrm{PS}_{36}-\mathrm{b}-\mathrm{PAA}_{125}$ were dissolved at $60^{\circ} \mathrm{C}$ in $3 \mathrm{~mL}$ of dioxane (Sigma-Aldrich) for 2 days. Then, $2 \mathrm{~mL}$ of dry toluene were added to the solution, to obtain a $1 \mathrm{~g} . \mathrm{L}^{-1} \mathrm{PS}_{36}-\mathrm{b}-\mathrm{PAA}_{125}$ solution in $60 \% / 40 \%$ dioxane/toluene mixture. ${ }^{29,30,32} \mathrm{~A}$ Teflon Langmuir through was filled with $0.1 \mathrm{M} \mathrm{NaCl}$ solution in MilliQ water, and the $\mathrm{PS}_{36}-\mathrm{b}-\mathrm{PAA}_{125}$ solution was spread at the air/water interface (21.4 $\mathrm{nm}^{2}$ per copolymer molecule). Before any measurement, the solvent was allowed to evaporate for 30 min. Surface pressure-area isotherms were measured at a compression rate of $5 \mathrm{~mm} / \mathrm{min}$, before the $\mathrm{PS}_{36}-\mathrm{b}_{-}-\mathrm{PAA}_{125}$ 
molecules were transferred on the PS-coated surfaces at concentrations between 0.05 and 0.07 polymer chains $/ \mathrm{nm}^{2}$. The surfaces were then dried using dry filtered nitrogen gas, and heated at $110^{\circ} \mathrm{C}$ for $15 \mathrm{~min}$ to improve the anchoring of the $\mathrm{PS}_{36^{-}}$ b-PAA ${ }_{125}$ molecules on the PS film. ${ }^{32}$ A similar preparation method, replacing the quartz crystal by silicon wafers, was used for the AFM experiments. A typical Langmuir isotherm showing the transfer conditions used in this work is presented in the supporting information (Fig. S1).

In a second approach, disulfide functionalized poly(acrylic acid) was used to produce low-density PAA layers on gold surfaces via spontaneous self-assembly. Gold-coated quartz crystals were immersed in $1 \mathrm{mg} / \mathrm{ml}\left(\mathrm{PAA}_{47}\right)_{2} \mathrm{~S}_{2}$ solutions in ethanol during $48 \mathrm{~h}$, to enable the chemical grafting of the thiol modified polymers on the surface an attain high coverage. After that period, the surfaces were rinsed with pure ethanol to remove non-grafted material, and dried with dry nitrogen. For AFM experiments $\left(\mathrm{PAA}_{47}\right)_{2} \mathrm{~S}_{2}$ was grafted on atomically flat gold surfaces prepared by mica-templating, as described by Hegner and coworkers. ${ }^{33}$

\section{Atomic Force Microscopy, AFM}

Morphology of the $\mathrm{PS}_{36}-\mathrm{b}-\mathrm{PAA}_{125}$ and $\left(\mathrm{PAA}_{47}\right)_{2} \mathrm{~S}_{2}$ films immersed in water and tip-polymer brush interaction forces were determined by Atomic Force Microscopy in soft-contact or peak-force mode (Multimode 8 , Bruker) using Si tips on triangular silicon nitride cantilevers (Bruker, SNL10-B). Only horizontal flattening has been used to treat the measured raw data.

The interaction between the AFM tip and the PAA brushes as a function of the relative tip-substrate separation was measured by monitoring the cantilever deflection during approachseparation cycles of the tip-polymer brush pair. We considered the tip to be in contact with the surface when the measured deflection increased steeply (and linearly) with surface displacement; we have followed the common practice of using the data on this linear response range to calibrate the response of the photodiode for each force curve in order to obtain the relative surface-tip separations. ${ }^{34}$

\section{Quartz Crystal Microbalance with dissipation monitoring, QCM-D}

The conformation of PAA brushes and its response to changes in $\mathrm{pH}$ or salinity was measured in a commercial quartz crystal microbalance (QCM-D E1, Q-Sense). QCM is becoming an increasingly popular tool in studying the properties of thin films, to some extent because it is relatively easy to implement. As the frequency and width of resonance peaks of quartz crystals are modified by its near environment, if a crystal is decorated with a thin layer its mechanical resonances may be noticeably modified. Small changes in the configuration of the adsorbed layer (e.g. thickness, hydration) will directly translate into significant variation in the frequencies and widths of the resonance peaks of the crystal. ${ }^{35}$ Thus, by studying the resonance of a quartz crystal coated with a polyelectrolyte (PE) layer, changing properties of the coating can be monitored. The principles of the technique have been extensively described in
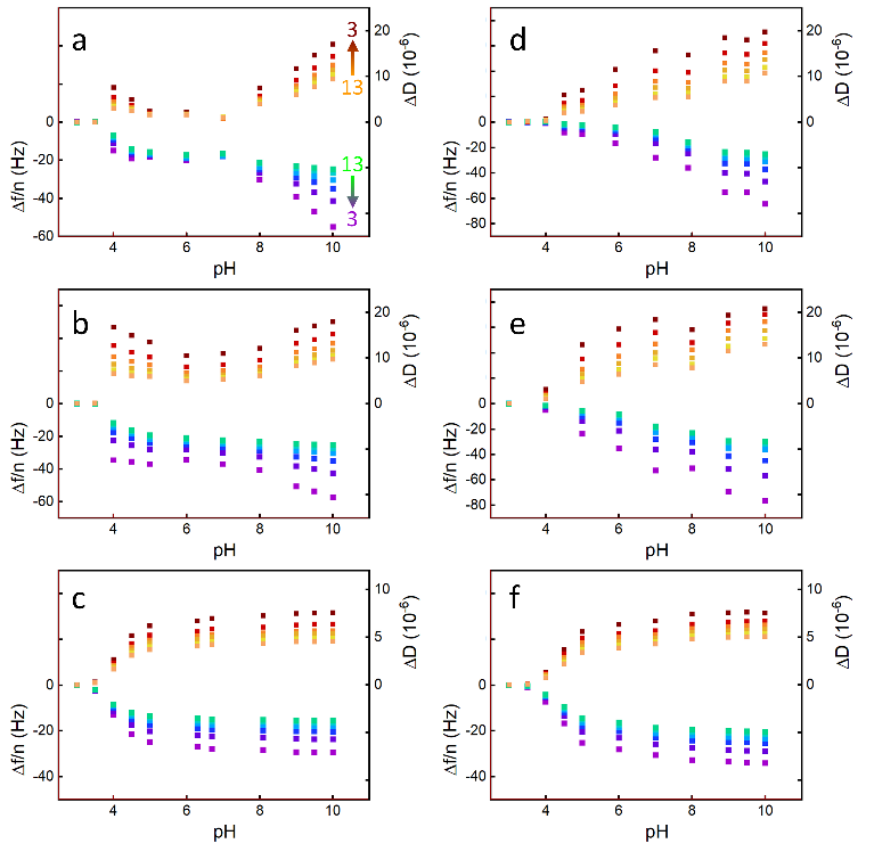

Figure 1.QCM-D: $\mathrm{PS}_{36}$-b-PAA 125 film on a PS-coated Quartz crystal. $\Delta f$ (negative values) and $\triangle D$ (positive values) for the different odd-harmonics (as indicated in a). $\mathrm{PS}_{36}$-b$\mathrm{PAA}_{125}$ grafting density: a),b),c) $\mathrm{PS}_{36}-\mathrm{b}-\mathrm{PAA}_{125} 0.05$ chains $/ \mathrm{nm}^{2} \mathrm{~d}$ ),e),f) 0.07 chains $/ \mathrm{nm}^{2}$. a),d) No salt added. b) $[\mathrm{KCl}]=0.6 \mathrm{mM}$. e) $[\mathrm{KCl}]=2 \mathrm{mM} . \mathrm{c}), \mathrm{f})[\mathrm{KCl}]=500 \mathrm{mM}$

the literature. ${ }^{36}$ Briefly, the resonance frequency $f$ of a quartz resonator is measured; a change in the effective mass of the resonator due to material adsorption translates into a variation in the resonance frequency, $\Delta f$. In addition, the damping of the oscillation of the crystal was measured, and used to calculated the "dissipation factor", $D$, defined as the inverse of the quality factor of the resonance peak. ${ }^{37} \mathrm{~A}$ large value of $D$ indicates quickly decaying oscillations of the crystal, which is observed for thicker and non-rigidly attached layers. In a QCM-D experiment, the measured $\Delta f$ and $\Delta D$ can be related to the thickness and viscoelastic properties of the material adsorbed on the quartz crystal by using adequate models. ${ }^{36}$

We used 5.0 $\mathrm{MHz}$ quartz resonators with gold electrodes, which were coated with PAA by the two methods described above. At the beginning of each experiment the coated resonator was placed in the cell and immersed in Milli-Q water adjusted to the $\mathrm{pH}$ and salt concentration to be studied for at least 30 minutes, until a stable baseline was established. This enabled the system to thermally equilibrate. The effect of $\mathrm{pH}$, concentration of supporting salt $(\mathrm{KCl})$ and grafting density $d$ on polymer conformation was investigated by measuring the response of the odd harmonics ( $n=3$ to $n=13$ ).

For both, QCMD and AFM experiments, $\mathrm{pH}$ was varied between ca. 2.7 and 10, by adding the necessary amounts of $\mathrm{HCl}$ and $\mathrm{KOH}$, without using buffer solutions. Ionic strength was tuned by adding adequate amounts of $\mathrm{KCl}$.

\section{Results and discussion}

Quartz Crystal Microbalance 


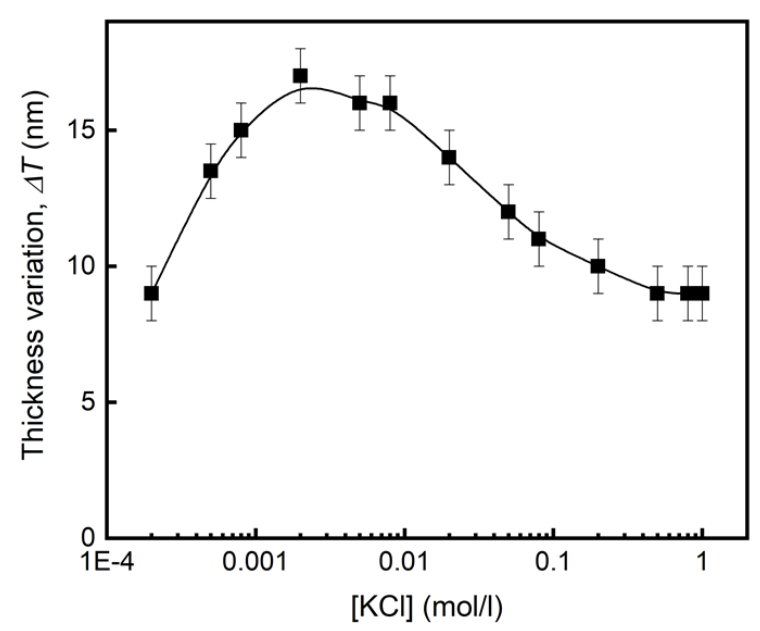

Figure 2. Relative thickness variation of a $\mathrm{PS}_{36}-\mathrm{b}-\mathrm{PAA}_{125}$ film, calculated from changes in $\Delta f$ and $\Delta D$, as described in the text. $\mathrm{PS}_{36}$-b-PAA $\mathrm{P}_{125}$ monolayer transferred at $\Pi=0.2 \mathrm{mN} / \mathrm{m}\left(0.05\right.$ chains $\left./ \mathrm{nm}^{2}\right)$. $\mathrm{pH}$ 5.5. Line connecting the data points is a guide to the eye

We have explored the influence of $\mathrm{pH}$, ionic strength $I$ and grafting density $d$ on the configuration of the polymer brushes. We decided against using buffer solutions for $\mathrm{pH}$ control, to have an accurate quantification of $I$. Common buffer solutions involve using multivalent salts with a significant impact on I which is difficult to quantify. Thus, $\mathrm{pH}$ variation was achieved by adding controlled amounts of $\mathrm{KOH}$ or $\mathrm{HCl}$; we minimized the exposure of the different solutions to the atmosphere and the time elapsed between solution preparation and data acquisition to avoid spurious change in $\mathrm{pH}$ (due, e.g., to $\mathrm{CO}_{2}$ dissolution, particularly when working close to neutrality).

The measured changes of crystal resonance frequency $\Delta f$ and dissipation factor $\Delta D$ measured with respect to the values at $\mathrm{pH}$ 3 are presented in Figure 1. The exploration of QCM-D raw data is very revealing; as can be observed in the Figure, the variation of $\Delta f$ and $\Delta D$ don't always show a monotonic behavior with the $\mathrm{pH}$ of the environment. In particular, for low grafting densities and low salinities (Fig. 1a-b), the absolute values of both $\Delta f$ and $\Delta D$ first increase, then decrease and finally increase again when $\mathrm{pH}$ is augmented. On the contrary, for larger grafting densities or larger salt concentrations (Fig. 1c-f) the absolute value of both $\Delta f$ and $\Delta D$ monotonically increase with $\mathrm{pH}$.
It is clear that the measured values of $\Delta f$ and $\Delta D$ are quite sensitive to the state of the polymer brush, which is modified by the physicochemical environment. However, translating the variation in $\Delta f$ and $\Delta D$ into physically relevant parameters characterizing the polymer layer can be challenging, often requiring the description of the polymer coating via oversimplified models. In this work, we have used the small load approximation, as described by Johansmann and others. ${ }^{35}$ The adsorbed layer is modelled as a homogeneous film of a given thickness, $T$, with complex shear modulus $G^{*}=G^{\prime}+i G^{\prime \prime}$, where $G^{\prime}$ and $G^{\prime \prime}$ are the storage and loss modulus of the film, respectively. As the QCM operates at relatively large frequencies ( $\mathrm{MHz}$ range), we have assumed for data fitting a simple power-law dependence of both components of the modulus with frequency $\omega$ (equation 2)

$$
G^{\prime}(\omega)=G^{\prime}\left(\omega_{0}\right) .\left(\omega / \omega_{0}\right)^{\alpha} ; G^{\prime \prime}(\omega)=G^{\prime \prime}\left(\omega_{0}\right) \cdot\left(\omega / \omega_{0}\right)^{\beta}
$$

where $\alpha$ and $\beta$ are the exponent describing the frequency dependence of $G$ and $\omega_{0}$ is a suitable reference frequency (we have chosen $5 \mathrm{MHz}$, the fundamental resonance frequency of the crystals). To reduce the number of fitting parameters, we have assumed that the viscosity of the film $\eta$ was frequency independent (i.e., $\beta=1$; by definition $\eta=G^{\prime \prime} / \omega$ ). Thus, four parameters (apparent film thickness $T$, complex shear modulus $G^{*}$ and exponent $\alpha$ ) are adjusted to fit 12 measured variables ( $\Delta f$ and $\Delta D$ for the odd harmonics 3 to 13 ). Unphysical results of the fit (e.g. negative values for the moduli, unrealistic film thickness or large values of $\alpha$ ) were systematically rejected. Despite of all the approximations involved, we found a very good fit of the measured data by this approach. In general, the regression results appeared quite robust; a smooth variation in all fitted variables was observed with $\mathrm{pH}$, although we only discuss here the obtained apparent film thickness (typical results of data fit are presented in the SI, Fig. S2).

The measured variation of the apparent film thickness with concentration of indifferent salt $(\mathrm{KCl})$ at $\mathrm{pH} 5.5$ is presented in Figure 2. As the polymer is deposited on the quartz crystal before the experiment, only relative variations of its properties are accessible. The relative thickness presented in this and following plots was calculated considering the state at $\mathrm{pH} 3$ and no added salt as zero. As can be observed in the figure, the
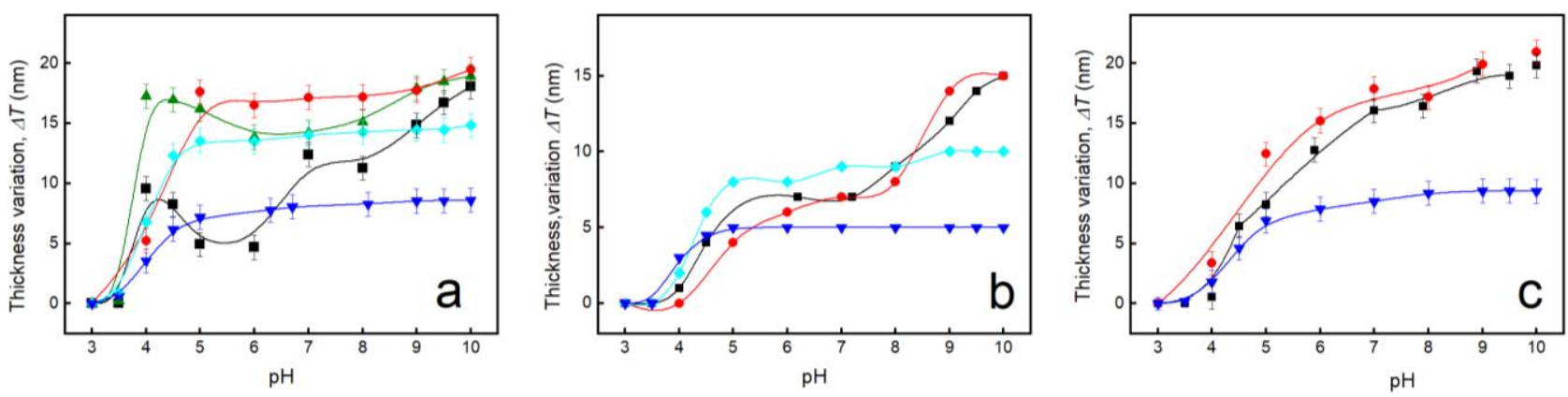

Figure 3. Relative thickness variation of a $\mathrm{PS}_{36}-\mathrm{b}-\mathrm{PAA}_{125}$ film vs $\mathrm{pH}$, calculated from changes in $\triangle f$ and $\Delta D$ of the coated quartz crystal, as described in the text. $\mathrm{PS}_{36}$-b-PAA ${ }_{125}$ density (a) 0.05 chains $/ \mathrm{nm}^{2}$; (b) 0.06 chains $/ \mathrm{nm}^{2}$; (c) 0.07 chains $/ \mathrm{nm}^{2}$. Black squares: no added salt. Green triangles: [KCl] $=0.6 \mathrm{mM}$. Red circles: $[\mathrm{KCl}]=2 \mathrm{mM}$. Cyan rhombi: $[\mathrm{KCl}]=50 \mathrm{mM}$. Blue inverted triangles: $[\mathrm{KCl}]=500 \mathrm{mM}$. Lines connecting the data points is a guide to the eye 

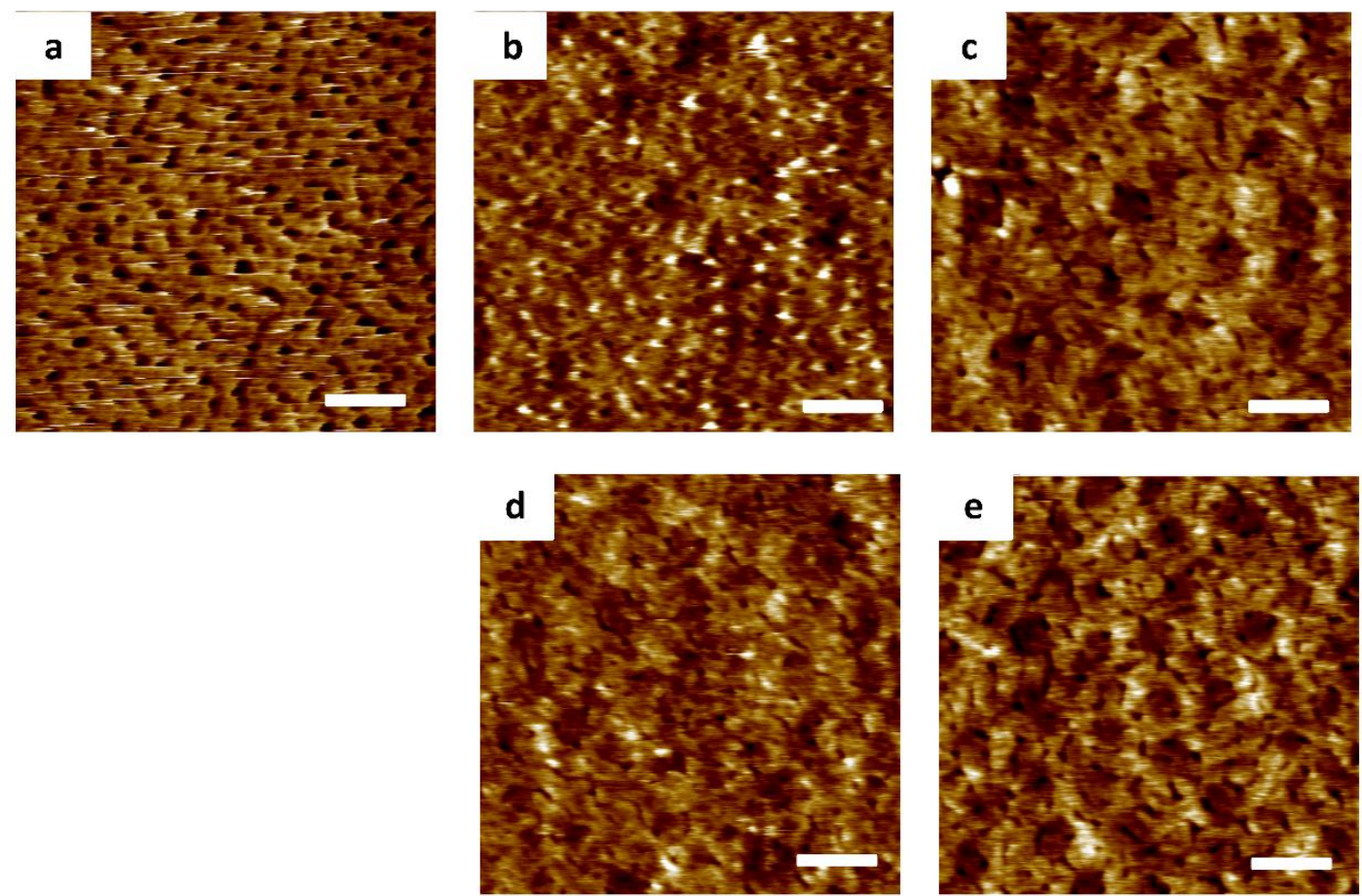

Figure 4. AFM soft contact micrographs in liquid environment of $\mathrm{PS}_{36}-b-\mathrm{PAA}_{125}$ coated surfaces; 0.05 chains $/ \mathrm{nm}{ }^{2}$. First row: $[\mathrm{KCl}]=0.6$ $\mathrm{mM}$. Second row: $[\mathrm{KCl}]=2 \mathrm{mM}$ a) $\mathrm{pH}=3$. b) $\mathrm{pH}=5$. c) $\mathrm{pH}=10$. d) $\mathrm{pH}=7$. e) $\mathrm{pH}=10$ (scale bars $200 \mathrm{~nm}$ ).

thickness of the annealed PE brush is strongly dependent on the ionic strength: it first increases and then decreases with salt concentration. The nonmonotonic change of weak polyion brushes with ionic strength has been predicted ${ }^{9,2}$ and reported in a number of experimental studies. ${ }^{29,38,39,40,41}$ At low ionic strengths the protons remain close to the polymer molecules, increasing the local $\mathrm{pH}$ and hindering ionization of the polyacid. As the salinity is increased, the protons are replaced by the potassium counterions. Consequently, the local $\mathrm{pH}$ and the effective degree of ionization of the PE increase at higher values of $l$, promoting the swelling of the grafted polymer chains (osmotic brush regime). Finally, at larger salinities the electrostatic repulsion between ionized monomers is largely screened (salted brush regime), effectively promoting the shrinking of the PE brush. Thus, it is clear that other than $\mathrm{pH}$, configuration of weak polyacids can also be tuned by the ionic strength. This behaviour, expected for a weak PE, evidences their sensitivity to their physicochemical environment. Nevertheless, both the QCM data analysis and the mean field description just outlined assume a laterally homogeneous brush layer with a well-defined thickness. As will be described later, this assumption is not always valid.

The variation of apparent brush thickness with $\mathrm{pH}$ at different concentrations of salt and grafting densities are presented in Figure 3. As could be anticipated, for the lower $d$ value investigated the changes in the calculated thickness map the singular behaviour of the measured $\Delta f$ and $\Delta D$ (0.05 chains $/ \mathrm{nm}^{2}$; Fig. 3a): at low salt concentrations, a non-monotonic dependence of $\Delta T$ with $\mathrm{pH}$ is observed. The effective thickness of the polymer brush increases at low $\mathrm{pH}$, stays constant or decreases at intermediate $\mathrm{pH}$ values (defining an intermediate regime that can extend up to $4 \mathrm{pH}$ units at low I) and finally reaches a second plateau at more basic $\mathrm{pH}$ conditions. On the contrary, at larger ionic strengths or grafting densities (Fig. 3c) the apparent thickness increases monotonically with the environmental $\mathrm{pH}$ for all I investigated, driven by the increasing contribution of the electrostatic repulsion between the monomers. This is due to the continuous increase of the degree of ionization of the chains with increasing $\mathrm{pH}$. When the polyion ionization occurs monotonically (at sufficiently large l), the transition appeared to be more gradual and at higher $\mathrm{pH}$ values for larger grafting densities (cf. Fig. S3b). The intermediate regime observed also depends on the brush grafting density; its extension is substantially reduced at the intermediate condition ( 0.06 chains $/ \mathrm{nm}^{2}$, Fig. $3 \mathrm{~b}$ ) and completely disappeared at the largest density investigated ( 0.07 chains $/ \mathrm{nm}^{2}$; Fig. 3c). The results presented in Figure 3 correspond to the PAA brush produced by the L-B method. Qualitatively similar results to the low-density case were found for the self-assembled polymer brush, as can be observed in the Fig. S3a (supporting information). However, as the molecular weight of the PAA chains are shorter in this case, a less significant change in effective thickness upon swelling is observed. For instance, in pure water no variation of $T$ is observed below $\mathrm{pH}$ ca. 8

\section{Atomic Force Microscopy: $\mathrm{PS}_{36}-\mathrm{b}-\mathrm{PAA}_{125}$}

We have imposed the condition of a laterally homogeneous polymer brush with well-defined thickness in the QCM data analysis. To validate this hypothesis we have used AFM in liquid environment, in order to assess the local morphology of the PE brush in different conditions of $\mathrm{pH}$ and $I$. Typical results are presented in Figures 4, 5, S5 and S6. The images reported in this section were measured in soft-contact mode. In this mode, a 
feed-back loop is used to impose a particular cantilever deflection while scanning, which translates into a constant tipsurface interaction force while operating. As it is customary in imaging soft surfaces, we always imposed the minimal normal force that still allowed a stable tip scanning, to avoid modification of the polymer layer by the rastering tip. However, as the charge density $\sigma$ of both tip and polymer layer are $\mathrm{pH}$ dependent, the interaction between them changed significantly for different operational conditions. At acid $\mathrm{pH}$ the polyion is practically free of charge and $\sigma$ of the tip is substantially reduced. In this condition the tip-substrate long range interaction is dominated by dispersive attractive forces, and the AFM images are measured when the repulsive steric forces dominate (with the tip and the surface effectively in contact). On the contrary, at basic $\mathrm{pH}$ conditions both the tip and the brush are negatively charged, and there is a long-range electrostatic repulsive force between them. In this condition surface topography is imaged in soft-contact mode by establishing the cartography of this long-range repulsion, without bringing the tip in real contact with the surface. Typical tip-substrate interaction force profiles are presented in the supporting information (Fig. S4)

As can be observed in Figures 4 and $5, l, \mathrm{pH}$ and $d$ have all significant influence on the morphology observed. No data treatment (other than flattening, to correct for the unavoidable slope difference between the surface and the scanning direction) has been applied. At higher grafting densities (Fig. 5 and S5) there are no substantial changes on the AFM images obtained with changing $\mathrm{pH}$, other than some increase on the typical size of the observed features, which may be identified to polymer swelling. On the contrary, the effect of $\mathrm{pH}$ on surface topography at conditions of low $d$ is more evident.

i) Acid pH (Fig.4a). In this condition a homogeneous distribution of holes 3-4 $\mathrm{nm}$ deep is observed in the polymer layer. This holes were not observed at higher grafting densities (Fig. 5a and S5a). Some streaks are observed on the images (cf. Fig 4a), which are likely due to the tip-surface adhesive interaction. Increasing / by adding $\mathrm{KCl}$ did not induce significant modifications on the observed topography.

ii) Intermediate $\mathrm{pH}$. (Fig. 4b, S6) At the conditions corresponding to the intermediate region found by QCM-D, the
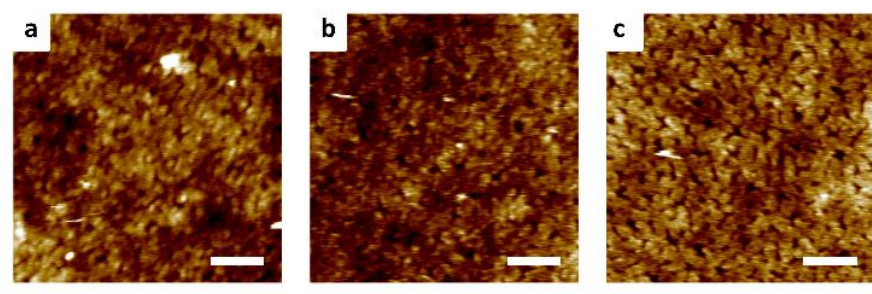

Figure 5. AFM soft contact micrographs in liquid environment of $\mathrm{PS}_{36}-b-\mathrm{PAA}_{125}$ coated surfaces; 0.07 chains $/ \mathrm{nm}^{2}$. [KCl] $=0.6 \mathrm{mM}$. a) pH=3. b) pH=7. c) pH=10 (scale bars $200 \mathrm{~nm}$ )

obtained AFM images were strongly dependent on both I and $d$, with a distinctive heterogeneous topography. Small zones that appear to be above the base level of the brush (white circular regions in the Figure) are regularly distributed on the surface. These regions appear to be of $20 \mathrm{~nm}$ lateral size and 2-5 nm in height (above the rest of the surface). However, both quantities must be taken with care: the measured lateral size is biased by the tip radius, and the height is extremely dependent on the applied normal force (set point). The typical distance between these asperities is ca. $40 \mathrm{~nm}$, which may be compared with the value of the Debye length which determines the range of the electrostatic interaction (12 $\mathrm{nm}$ for the case of Fig. 4b). Significantly, these regions are almost or completely absent at larger I (Fig. 4d) or $d$ values (Figs. 5b and S5b).

iii) Basic $\mathrm{pH}$. At higher $\mathrm{pH}, d$ or $I$ values (i.e., at $\mathrm{pH}$ above the intermediate region for low $d$ ) a homogeneous topography is again observed in the AFM images in all cases. Given that in this conditions there is a strong long-range electrostatic repulsion between the tip and the substrate due to the increasing ionization of the polyions (cf. Fig. S4) the presence of streaks and flickering in the images is substantially reduced. The contrast in morphology between intermediate and basic $\mathrm{pH}$ is very marked, as can be observed in the larger scale images presented in Figure $\mathrm{S} 6$.

\section{Atomic Force Microscopy: $\left(\mathrm{PAA}_{47}\right)_{2} \mathrm{~S}_{2}$}

The two main features observed in the exploration of the changes in the brush of $\mathrm{PS}_{36}-\mathrm{b}-\mathrm{PAA}_{125}$ with $\mathrm{pH}$ (non-monotonic swelling with increasing $\mathrm{pH}$ and apparent heterogeneity) might
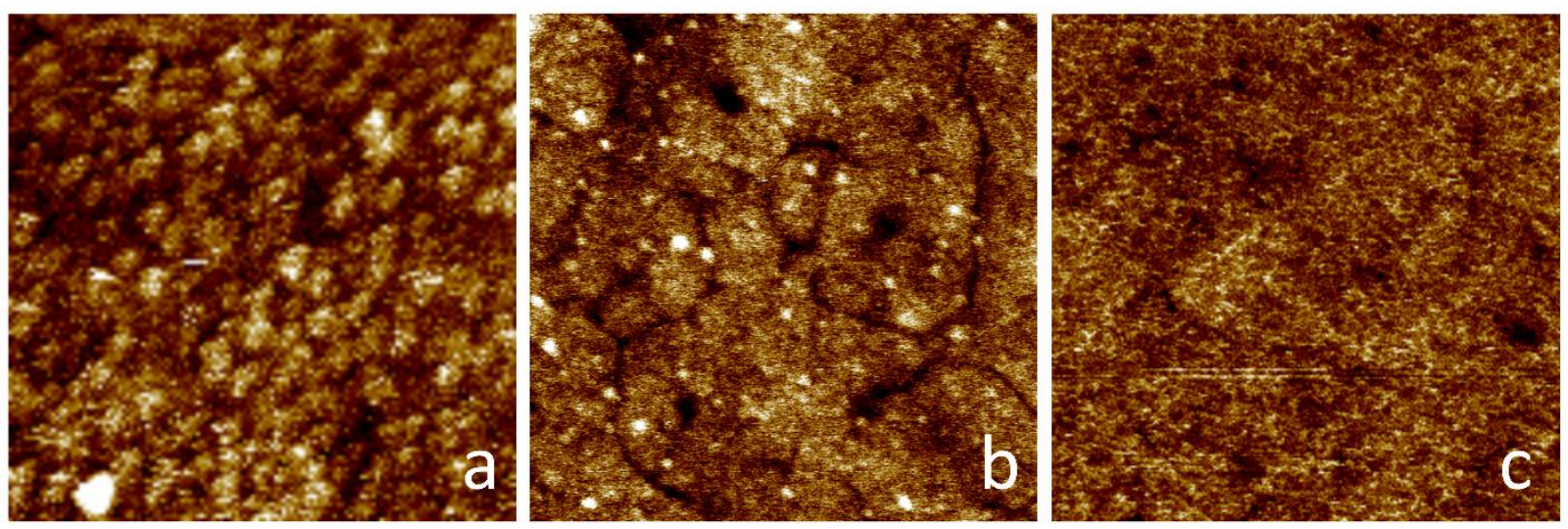

Figure $6.500 \mathrm{~nm} \times 500 \mathrm{~nm}$ peak-force mode height AFM micrographs of a $\left(\mathrm{PAA}_{47}\right)_{2} \mathrm{~S}_{2}$ self-assembled film measured in water. a) pH 2.8; b) pH 6.0; c) pH 10.4. For each case, the maximum applied force (set point) was fixed to the minimum value for which a stable image could be obtained. The colour scale spans $6 \mathrm{~nm}$ in all images 
be related to the preparation method of this system, and in particular to the presence of a hydrophobic PS sublayer for polymer anchoring. To evaluate the robustness of the results, we performed similar AFM experiments with the PAA brush self-assembled on gold. In these experiments we recurred to the PeakForce mode, to reduce the tip-polymer interaction while scanning as much as possible. In this mode an approachretraction cycle (superimposed to a fast small amplitude oscillatory motion, frequency $2 \mathrm{kHz}$, amplitude $100 \mathrm{~nm}$ ) is performed on each point of the sample to be imaged, while controlling the maximum cantilever deflection. By these means, the disruptive effect of the tip is minimized. Typical PeakForce mode height micrographs are presented in Figure 6. At low pH $(\mathrm{pH}<3)$, self-organization in the polymer layer could be observed: the surface appears to be fully covered by micelle-like elements (30-50 nm lateral size). At basic pH (> 8.5, Fig. 6c) a rather homogeneous aspect can be observed. The situation was more complicated at intermediate $\mathrm{pH}$ values (5-6). As observed with the LB deposited brush, features between 2 and $5 \mathrm{~nm}$ tall regularly distributed on a homogeneous layer were often observed (Fig. 6b). In some cases larger islands or coexistence of small and larger islands were also observed (Fig. S7). Often the larger islands disappeared after scanning at sufficiently large set-point (tip-substrate interaction; cf. Fig. S8). The fading of these features was scanning-induced, even though low set points were typically applied (maximum applied force below $200 \mathrm{pN}$ ). Interestingly, islands were typically observed in a 'fresh' (not previously imaged) spot, few hours after $\mathrm{pH}$ exchange. On the other hand, we couldn't detect re-emergence of the larger islands in a previously scanned area. Thus, even though they were long lasting, it is not clear that the taller features correspond to the equilibrium state of the polymer brush. The fact that they were easily and irreversibly perturbed by the scanning tip suggests that they correspond to metastable, kinetically frozen aggregates. This historydependent aggregation process is likely to be related to hysteretic properties in PAA brush layers, recently reported by several groups. ${ }^{42,43}$

These AFM results indicates, once again, a complex dependence on $\mathrm{pH}$ for the PAA brush. However, the imaging conditions are different for the different $\mathrm{pH}$ conditions, mainly because the tipsubstrate interaction is a strong function of $\mathrm{pH}$, as described above. Thus, it is revealing to directly investigate the effect of $\mathrm{pH}$ on this interaction. We have collected a large number of force curves at different $\mathrm{pH}$ conditions, with special attention to the long-range interaction force, which provides information about the state of the surface without direct tip-substrate contact. Typical results measured on approach are presented in Fig. 7. Each force curve represented corresponds to the average of 20 individual force profiles measured in different spots on the surface. These results were robust; similar results were obtained in two $\mathrm{pH}$ cycles with the same surface, and with two independently prepared PAA brushes. As can be observed in the figure, a non- monotonic dependence of interaction force with $\mathrm{pH}$ is apparent. At $\mathrm{pH} 3$, when the PAA chains are mostly nonionized, there is only a short range, repulsive interaction of steric origin between the tip and the surface. On the contrary,
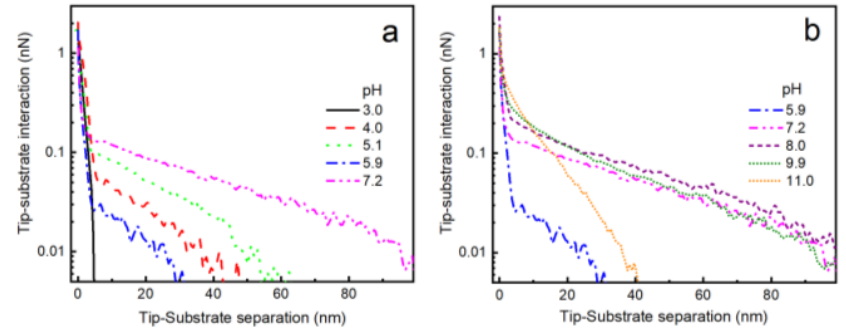

Figure 7. Normal Interaction force between the AFM silicon tip and the selfassembled film of $\left(\mathrm{PAA}_{47}\right)_{2} \mathrm{~S}_{2}$ measured on approach under different $\mathrm{pH}$ conditions, as indicated

for more basic $\mathrm{pH}$ conditions, a clear long-range repulsive interaction is observed. The exponential decay of this repulsive force strongly hints of its electrostatic origin, due to the negative charge in both the AFM Si tip and the PAA brush. The fitted decay-length of the electrostatic repulsion matches well with the Debye length that could be expected for the ionic strength of the different $\mathrm{pH}$ conditions (cf. Table S1), except for the case of conditions close to neutrality $(\mathrm{pH} 8)$. In this case, an extremely low ionic strength (ca. $10^{-6} \mathrm{M}$ ) could be anticipated. However, this is not attainable, given that we are using a strong acid $(\mathrm{HCl})$ and a strong base $(\mathrm{KOH})$ to tune the $\mathrm{pH}$. As can be expected, the decay length of the force at $\mathrm{pH} 11$ is significantly shorter than at lower $\mathrm{pH}$ conditions, due to the increased ionic strength in this case. The magnitude of the force is directly determined by the ionization degree of the brush. However, its progression with increasing $\mathrm{pH}$ is non-monotonic: it increases from $\mathrm{pH} 4$ to 5 , and then shows a marked decrease at $\mathrm{pH} 5.9$, to then increase monotonically from $\mathrm{pH} 6$ to 11 . The repulsive force at short separations is considerably larger at the most basic conditions, signalling a more complete PAA ionization. The progression of the measured force with increasing $\mathrm{pH}$ correlates very well with the non-monotonic swelling observed by QCM (Fig. 3a), with a clear minimum at intermediate $\mathrm{pH}$ values.

Combined AFM results point to an analogous behaviour of the two brushes investigated, with heterogeneous morphology at conditions of low $I$ and intermediate $\mathrm{pH}$, re-entrant swelling at low $I$, and polymer self-assembly (holes or pinned micelles) at low $\mathrm{pH}$. As mentioned above, the effect of the PS adlayer on the LB polymer brush, which will be more important at low grafting densities, cannot be completely discarded. Nevertheless, the fact that similar results were obtained with the self-assembled PE brush on gold surfaces, strongly supports the general relevance of the non-monotonic behaviour of low-density PE brushes observed by us.

\section{Discussion}

Re-entrant $\mathrm{pH}$ swelling. As mentioned above, the nonmonotonic behaviour of the weak polyion with $I$ described above has been theoretically predicted and experimentally

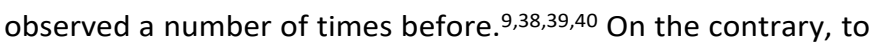
the best of our knowledge the unusual re-entrant swelling with increasing $\mathrm{pH}$ reported here is unprecedented. The observed variation of the effective thickness of the PE brush at low 
grafting densities (Fig. 3a) and the non-monotonous evolution of the tip-substrate repulsion observed by AFM (Fig. 7) are both related to the changing degree of ionization of the PE brush at low salinities. Rather counterintuitively, increasing the bulk pH does not necessarily imply a larger degree of ionization of the acrylic acid chains close to neutral $\mathrm{pH}$.

Several processes are probably the reason of this nonmonotonic evolution. First, in pure water, without addition of supporting electrolyte, increasing (decreasing) $\mathrm{pH}$ in the acid (basic) region comes along with a decrease of ionic strength $I$, which reduces the screening of electrostatic interactions. The enhanced unfavourable interaction between nearby charged monomers can be partially compensated by a shift in the acidbase equilibrium (charge regulation), reducing the concentration of charges. But even in the absence of a shift of $p K_{a}$, the local $\mathrm{pH}$ in the brush region, which determines the ionization of the polyion, can be substantially more acidic than the bulk value because of the required local electroneutrality. For this reason, increasing bulk $\mathrm{pH}$ while reducing $I$, may lessen the ionization degree of the polyacid. This was clearly described by Wolterink and coworkers in a theoretical study of starbranched PEs. ${ }^{6}$ They described how at low I a plateau can be observed for intermediate $\mathrm{pH}$ values in the titration curve (PE ionization degree vs $\mathrm{pH}$ ). This plateau is present for small number of arms, which can be linked to the case of low grafting density in this study, and disappears at larger number of arms, which may be associated to a larger grafting density.

\section{In-plane heterogeneity}

We observe clear evidences of heterogeneity in PAA brushes, mainly at low grafting densities. Two cases can be identified. At low $\mathrm{pH}$, when the polymer chains are essentially protonated (neutral), microstructuration could be identified, with the formation of holes (Fig. 4a) or aggregates (Fig. 6a) in the polymer layer. As discussed in the introduction, this selfassembly may be driven by the attractive interaction between the grafted chains: limited segregation leading to the formation of spherical micelles and holes has been predicted by a number of theoretical studies. ${ }^{17,21,22}$ The required ingredients are an attractive interaction between the uncharged segments counteracted by the limited mobility of the grafted chains. Even though water is a good solvent for isolated PAA chains, ${ }^{44}$ it is conceivable that the solvent quality decreases at high polymer concentrations. It is also likely that inter-chain hydrogen bonding (a close-range attractive interaction) promotes PAA aggregation, favouring the observed self-assembly. The difference in morphology between the two systems studied (holes vs. pinned micelles) is probably due to the different grafting density explored, which was likely to be lower in the case of the $\left(\mathrm{PAA}_{47}\right)_{2} \mathrm{~S}_{2}$ brush produced by self-assembly.

A different scenario appears at conditions of low $d$, low $I$ and intermediate $\mathrm{pH}$, where heterogeneous morphology of the PAA brush was observed (Figs. 4b, 6b, S6a). As mentioned in the introduction, experimental evidences of $\mathrm{PE}$ brush segregation are rather scarce. Block and Helm reported on the heterogeneity of adsorbed strong polyelectrolytes under conditions of low salinity; they observed the coexistence of flatly adsorbed polymer chains with brush regions of up to micrometer lateral size. ${ }^{45} \mathrm{Yu}$ and co-workers observed a marked lateral heterogeneity of strong polyion brushes due to multivalent counterion triggered intermolecular bridging. ${ }^{46}$ More relevant to the present study, Hur and co-workers reported lateral heterogeneity at the nanoscale level for weak polybase PDMAEMA brushes in deionized water or at low salinities, while the same polymer brushes appeared featureless at high ionic strength. ${ }^{27}$ They attributed this behaviour to the reduced degree of ionization of the grafted polybase compared to the monomer in bulk solution due to the higher local $\mathrm{pH}$ in the brush region. This limited ionization may drive polymer collapse or partial aggregation - restricted by the hindered mobility of the grafted chains- depending on the environmental conditions.

A number of theoretical works have suggested the possibility of heterogeneity in polyion brushes. Szleifer and co-workers developed a molecular theory for weak polyelectrolytes, and showed that grafted weak PE layers can be unstable at low salinity, particularly in bad solvent conditions; the stability of the layer was enhanced at high ionic strengths. ${ }^{8}$ In a follow-up work, it was shown that the balance between acid-base equilibrium and physical interactions (determined by solvent quality) can trigger microphase separation on grafted $P E$ layers. ${ }^{21}$ Zhulina and Borisov used mean field model and scaling arguments to study a PE brush grafted on an oppositely charged surface; the polymer chains were distributed in two populations, with some chains adsorbed on the adlayer and the rest stretched in the solvent. ${ }^{3}$ Nevertheless, the segregation was not observed in the absence of opposite charge on the surface. Shusharina and Linse reported the possibility of internal segregation in grafted brushes of an unchargedcharged diblock copolymer, with a population of chains near the surface and a second population stretching out of the surface, reducing the interchain electrostatic repulsion and the free energy cost of chain stretching. ${ }^{47}$ More relevant to the present study, in a theoretical study using a self-consistent-field model Lyatskaya and coworkers observed that the density profile of brushes of weak PEs are markedly dependent on the physicochemical environment. Remarkably, at low values of $I$ they observed a significant reduction in the degree of ionization of the polyions (due to the local reduction of $\mathrm{pH}$ described above) accompanied by a protruding (diluted) tail of charged chains, signaling a heterogeneous degree of ionization of the polyions. ${ }^{48} \mathrm{~A}$ broadly similar conclusion was drawn by Zhulina and Borisov using an analytical theory to describe annealed PE brushes; even though they assumed a lateral homogeneity of the grafted layer, they found that at low ionic strength the degree of ionization of the PE chains was nonhomogeneous, with weaker ionization closer to the grafting wall. ${ }^{10}$ Analogously, Klein Wolterink and coworkers found a bimodal distribution of degree of ionization for low $\mathrm{I}$, at $\mathrm{pH}$ close the $\mathrm{p} K_{a}$ of the polyacid, in the study of star-shaped PE discussed above. On the contrary, the distribution of net charges per arm becomes monomodal at higher ionization degrees. ${ }^{6}$

The non-homogeneous degree of ionization at low $I$ and $d$ at $\mathrm{pH}$ values close to the $\mathrm{p} K_{a}$ of the poly(acrylic acid) brushes studied 
in this work is likely to be the cause of the heterogeneous aspect observed by AFM. At low ionic strengths, polymer ionization in the brushes is hindered due to local $\mathrm{pH}$ reduction (as compared to the bulk values) and to greater intermolecular electrostatic repulsion. These effects acquire more relevance close to neutral $\mathrm{pH}$, when I can be extremely low, but will be substantially reduced at larger ionic strengths, due to the combined effect of enhanced screening of the electrostatic interaction and increase of local brush pH upon exchange of protons by other counterions.

There are several consequences of increasing grafting densities. First, the concomitant increasing of repulsive steric forces are responsible for the weaker $\mathrm{pH}$ dependence of the brush thickness and the apparent shift of polyion ionization to larger bulk $\mathrm{pH}$ values (small increase of apparent $p K_{a}$, Fig. S3b). In addition, increasing $d$ also impedes the reduction in ionization degree observed close to neutral conditions at low grafting densities. It is tempting to parallel this condition to the change of the dependence of the ionization degree with $\mathrm{pH}$ for starbranched polyelectrolytes when the number of branches increases, ${ }^{6}$ although the shift in apparent $\mathrm{p} K_{a}$ observed by us at the largest grafting densities is not large enough to unmistakeably explain the absence of ionization reduction at intermediate $\mathrm{pH}$ conditions. Finally, increasing $d$ reduces the self-assembly at low $\mathrm{pH}$ : at high coverage the chains are sufficiently close, and there is no need to form laterally inhomogeneous structures to 'activate' a favourable interaction (e.g. inter-chain hydrogen bonding).

\section{Conclusions}

We have studied the influence of environmental conditions on apparent thickness and structure of brushes of grafted poly(acrylic acid), a weak polyelectrolyte, by combining quartz crystal microbalance and Atomic Force Microscopy. We have observed a non-monotonic variation of the effective brush ionization degree with bulk $\mathrm{pH}$ at low grafting densities and ionic strength which results in re-entering brush swelling (swelling/de-swelling/swelling), and points to the difference between brush (local) and global $\mathrm{pH}$. Counterintuitively, the coupling between charge regulation (chemical equilibrium among the charged and uncharged polymer monomers) and local $\mathrm{pH}$ may end up in suppressed ionization with increasing $\mathrm{pH}$ at low concentrations of salt. In addition, we have found that the morphology of the polyelectrolyte brush can be heterogeneous in conditions of low salinity and ionization degree, probably as a consequence of the destabilizing effect of inter-chain electrostatic repulsion. In addition, AFM results show clear evidences of self-organization changes in the polymer brush driven by changes in environmental conditions at low $\mathrm{pH}$, pointing to the balance between short-range attractive interactions (possibly inter-chain hydrogen bonding) and repulsive electrostatic interactions. These effects, which highlight the complexity of the response of grafted annealed polyelectrolyte brushes, must be taken into account in the design of weak polyion-based smart surfaces.

\section{Conflicts of interest}

There are no conflicts to declare

\section{Acknowledgements}

We are grateful to Dr. Diethelm Johannsmann for critically reading the manuscript

\section{Notes and references}

1 M. A. C. Stuart, W. T. S. Huck, J. Genzer, M. Müller, C. Ober, M. Stamm, G. B. Sukhorukov, I. Szleifer, V. V Tsukruk, M. Urban, F. Winnik, S. Zauscher, I. Luzinov and S. Minko, Nat. Mater., 2010, 9, 101-13.

2 E. B. Zhulina, T. M. Birshtein and O. V. Borisov, Macromolecules, 1995, 28, 1491-1499.

3 E. B. Zhulina and O. V Borisov, Macromolecules, 1998, 31 , 7413-7422.

4 V. Sénéchal, H. Saadaoui, J. Rodriguez-Hernandez and C. Drummond, Langmuir, 2017, 33, 4996-5005.

5 M. Castelnovo, P. Sens and J.-F. Joanny, Eur. Phys. J. E, 2000, 1, 115-125.

6 J. Klein Wolterink, J. Van Male, M. A. Cohen Stuart, L. K. Koopal, E. B. Zhulina and O. V. Borisov, Macromolecules, 2002, 35, 9176-9190.

7 M. Ballauff and O. V. Borisov, Polymer (Guildf)., 2016, 98, 402-408.

P. Gong, J. Genzer and I. Szleifer, Phys. Rev. Lett., 2007, 98, 5-8.

R. Israëls, F. A. M. Leermakers and G. J. Fleer, Macromolecules, 1994, 27, 3087-3093. E. B. Zhulina and O. V Borisov, Langmuir, 2011, 27, 1061533.

11 V. A. Baulin and A. Halperin, Macromol. Theory Simulations, 2003, 12, 549-559. H. Jia, A. Wildes and S. Titmuss, Macromolecules, 2012, 45, 305-312.

13 R. Dong, M. Lindau and C. K. Ober, Langmuir, 2009, 25, 4774-4779.

14 K. Ehtiati, S. Z. Moghaddam, A. E. Daugaard and E. Thormann, Langmuir, 2020, 36, 2339-2348. E. B. Zhulina, T. M. Birshtein, V. A. Priamitsyn and L. I. Klushin, Macromolecules, 1995, 28, 8612-8620.

16 D. R. M. Williams, J. Phys. II, 1993, 3, 1313-1318.

17 S. K. Pattanayek, T. T. Pham and G. G. Pereira, J. Chem. Phys., 2005, 122, 214908.

18 E. Zhulina, C. Singh and A. C. Balazs, J. Chem. Phys., 1998, 108, 1175-1183.

19 D. J. Sandberg, J. Y. Carrillo and A. V Dobrynin, Langmuir, 2007, 23, 12716-12728.

20 J. M. Y. Carrillo and A. V. Dobrynin, Langmuir, 2009, 25, 13158-13168.

21 M. Tagliazucchi, M. O. de la Cruz and I. Szleifer, Proc. Natl. Acad. Sci. U. S. A., 2010, 107, 5300-5305.

22 M. Tagliazucchi, X. Li, M. Olvera de la Cruz and I. Szleifer ACS Nano, 2014, 8, 9998-10008. 
23 V. Koutsos, E. W. Van Der Vegte, E. Pelletier, A. Stamouli and G. Hadziioannou, Macromolecules, 1997, 30, 47194726.

24 D. Murakami, Y. Norizoe, Y. Higaki, A. Takahara and H. Jinnai, Macromolecules, 2016, 49, 4862-4866.

25 B. C. Choi, S. Choi and D. E. Leckband, Langmuir, 2013, 29, 5841-5850.

26 J. U. Günther, H. Ahrens, S. Förster and C. A. Helm, Phys. Rev. Lett., 2008, 101, 1-4.

27 J. Hur, K. N. Witte, W. Sun and Y. Y. Won, Langmuir, 2010, 26, 2021-2034.

28 S. Peng and B. Bhushan, RSC Adv., 2012, 2, 8557-8578.

29 E. P. K. Currie, A. B. Sieval, G. J. Fleer and M. A. C. Stuart, Langmuir, 2000, 16, 8324-8333.

30 E. P. K. Currie, A. B. Sieval, M. Avena, H. Zuilhof, E. J. R. Sudhölter and M. A. Cohen Stuart, Langmuir, 1999, 15, 7116-7118.

31 V. Sénéchal, H. Saadaoui, J. Rodriguez-Hernandez and C. Drummond, Faraday Discuss., 2017, 199, 335-347.

32 O. Hollmann and C. Czeslik, Langmuir, 2006, 22, 33003305.

33 M. Hegner, P. Wagner and G. Semenza, Surf. Sci., 1993, 291, 39-46.

34 H.-J. Butt, B. Cappella and M. Kappl, Surf. Sci. Rep., 2005, 59, 1-152.

35 D. Johannsmann, The Quartz Crystal Microbalance in Soft Matter Research, Springer International Publishing, Zurich, 2015.S8

36 I. Reviakine, D. Johannsmann and R. P. Richter, Anal. Chem., 2011, 83, 8838-48.

37 M. Rodahl and B. Kasemo, Rev. Sci. Instrum., 1996, 67, 3238-3241.

38 M. Biesalski, D. Johannsmann and J. Rühe, J. Chem. Phys., 2002, 117, 4988-4994.

39 T. Wu, P. Gong, I. Szleifer, P. Vlček, V. Šubr and J. Genzer, Macromolecules, 2007, 40, 8756-8764.

40 J. D. Willott, T. J. Murdoch, B. A. Humphreys, S. Edmondson, G. B. Webber and E. J. Wanless, Langmuir, 2014, 30, 1827-1836.

41 H. Zhang and J. Rühe, Macromolecules, 2005, 38, 48554860. J. L. Weidman, R. A. Mulvenna, B. W. Boudouris and W. A. Phillip, J. Am. Chem. Soc., 2016, 138, 7030-7039.

43 V. Yadav, A. V. Harkin, M. L. Robertson and J. C. Conrad, Soft Matter, 2016, 12, 3589-3599.

44 R. Schweins, J. Hollmann and K. Huber, Polymer (Guildf)., 2003, 44, 7131-7141.

45 S. Block and C. A. Helm, J. Phys. Chem. B, 2011, 115, 730113.

46 J. Yu, N. E. Jackson, X. Xu, B. K. Brettmann, M. Ruths, J. J. De Pablo and M. Tirrell, Sci. Adv., 2017, 3, 1-11.

47 N. P. Shusharina and P. Linse, Eur. Phys. J. E, 2001, 4, 399402.

48 Y. V. Lyatskaya, F. A. M. Leermakers, G. J. Fleer, E. B. Zhulina and T. M. Birshtein, Macromolecules, 1995, 28, 3562-3569. 